\title{
On the Difference between Two Widely Publicized Methods for Analyzing Oscillator Phase Behavior
}

\author{
Piet Vanassche, Georges Gielen and Willy Sansen \\ Katholieke Universiteit Leuven - ESAT/MICAS \\ Kasteelpark Arenberg 10, B-3001 Leuven, Belgium \\ piet.vanassche@esat.kuleuven.ac.be
}

\begin{abstract}
This paper describes the similarities and differences between two widely publicized methods for analyzing oscillator phase behavior. The methods were presented in [3] and [6]. It is pointed out that both methods are almost alike. While the one in [3] can be shown to be, mathematically, more exact, the approximate method in [6] is somewhat simpler, facilitating its use for purposes of analysis and design. In this paper, we show that, for stationary input noise sources, both methods produce equal results for the oscillator's phase noise behavior. However, when considering injection locking, it is shown that both methods yield different results, with the approximation in [6] being unable to predict the locking behavior. In general, when the input signal causing the oscillator phase perturbations is non-stationary, the exact model produces the correct results while results obtained using approximate model break down.
\end{abstract}

\section{INTRODUCTION}

Oscillators are key building blocks in almost all of today's communication systems. Their behavior, however, is often hard to analyze, since their functioning inherently relies upon nonlinear behavior. One of the most important characteristics of an oscillator is the way its phase responds to external signals. These external signals could be both unwanted, e.g. noise sources causing the phase noise, or wanted, e.g. sine waves injected for locking purposes.

In recent years, much research has been devoted to the analysis of oscillator phase behavior. Circuit simulation [10] offers the most simple solution. This approach, however, is time-consuming, especially for the Monte Carlo methods needed to deal with noisy inputs, and the results are not straightforward to interpret, obscuring analysis. More compact and insightful methods have been developed in both [3, 4, 8] and [6,9]. Both, quite popular, approaches model the oscillator phase behavior using a 1-dimensional differential or integral equation, which is much easier to solve than the full set of circuit equations. Using the original notation, [3] models the phase noise behavior as

$$
\frac{d \theta}{d t}(t)=\epsilon v(t+\theta(t)) n(t)
$$

while [6] starts from

$$
\theta(t)=\epsilon \int_{0}^{t} \Gamma(\tau) n(\tau) d \tau
$$

In both equations, $n(t)$ is an external source, noise or otherwise, $\theta(t)$ is the oscillator phase and $v(t), \Gamma(t)$ are functions depending upon the oscillator's topology. They are respectively called the perturbation projection vector (PPV) and the impulse sensitivity func- tion (ISF). The oscillator's output is then determined by

$$
V_{o s c}(t)=V_{s}(t+\theta(t))
$$

where $V_{o s c}(t)$ represents the actual oscillator output signal while $V_{S}(t)$ is a $T$-periodic solution of the input-free (noiseless) oscillator. Furthermore, $\epsilon \ll 1$ is a perturbation variable used to indicate the fact that $\theta(t)$ varies slowly as compared to the oscillator period $T$. Observing both equations (1) and (2), it is seen that, essentially, they differ only slightly from each other. The question hence rises whether one can expect any significant differences in results when comparing the phase behaviors they predict.

In this paper, we show that, for some classes of applications, the models (1) and (2) predict similar results, while for other classes, results are widely different. More precisely, it is shown that for $n(t)$ a stationary (noise) source, equations (1) and (2) will, up to 0 -th order in $\epsilon$, predict the same output phase noise. On the other hand, when $n(t)$ is no longer stationary, results diverge. A noteworthy example is given by an oscillator's injection locking behavior $[1,7]$. Here, the input source $n(t)=N \cos (2 \pi f t)$ is a single sine wave with $f$ near the oscillator's free-running frequency $f_{0}$. A harmonic oscillator, for example, will lock both its frequency and its phase to that of $n(t)$. It will be shown that the model (1) is capable of predicting this behavior, while (2) is not. Related to injection locking is the behavior of the phase differences $\Delta \theta$ within sets of coupled oscillators. Since the coupling effect can be considered as a mutual injection phenomenon, (1) yields correct results while (2) breaks down.

The main tool used for obtaining the results mentioned above is the averaging transformation as introduced in $[2,5]$. In this paper, we extend this transformation to its most general setting, allowing us to deal with both deterministic signals, white noise and colored noise. Using the averaging transformation, it becomes possible to separate the slow-varying components of the oscillator's phase behavior from the fast-varying ones. These slow-varying components typically contain those characteristics of the oscillator's behavior which are of greatest interest, like phase noise (wander) and locking.

The remainder of this paper is organized as follows. In section 2 , we briefly discuss both the origin and properties of both models (1) and (2) for describing oscillator phase behavior. Section 3 introduces averaging and its use for analyzing an oscillator's phase behavior. In section 4 , we apply this principle to analyze oscillator phase noise, showing that, under certain conditions, the results obtained from both model equations (1) and (2) are equivalent. Section 5 discusses the injection locking phenomenon and shows how both models predict widely different results. Finally, in section 6 , we demonstrate these differences with some circuit-level simulations. Conclusions are presented in section 7. 


\section{TWO DIFFERENT MODELS FOR OS- CILLATOR PHASE BEHAVIOR}

Two widely publicized methods for describing the behavior of the oscillator phase $\theta(t)$ are presented in [3] and [6]. In this section, we give a brief overview of both of them. We also discuss their properties, similarities and differences.

For a single input source $n(t)$, the method in [3] models the oscillator's phase behavior using the differential equation (1). This equation is derived using nonlinear perturbation techniques and is, up to first order in $\epsilon$, exact. For this reason, in what follows, we will call it the exact model. The quantity $v(t)$ is called the oscillator's perturbation projection vector (PPV) ${ }^{1}$. It is a $T$-periodic function modeling the way a particular input source affects the oscillator phase.

The method in [6], on the other hand, models the oscillator's phase behavior using the integral equation (2). Rewritten as a differential equation, this becomes

$$
\frac{d \theta}{d t}(t)=\epsilon \Gamma(t) n(t)
$$

This model was conjectured based upon physical reasoning. For this reason, in what follows, we will call it the approximate model. Here, the quantity $\Gamma(t)$ is a $T$-periodic function, called the impulse sensitivity function (ISF). It serves a similar role as the PPV, i.e. it models the way a particular input source affects the oscillator's phase.

Comparing equations (1) and (4), it is observed that both are almost alike. By considering their proposed numerical computations $^{2}$, it is easily verified that the PPV $v(t)$ equals the ISF $\Gamma(t)$. Furthermore, both of the oscillator phase behavior models (explicitly or implicitly) assume the phase $\theta(t)$ to be slow-varying compared to the oscillator period $T$, which is accomplished by setting $\epsilon \ll 1$. The only major difference is the fact that in equation (1), the phase $\theta$ also appears in the right-hand side, while this is not the case in equation (4).

The observed similarity between both model equations raises the question towards the difference in results they produce. Although (1) is in principle more exact, the appearance of $\theta$ in the right-hand side makes it more difficult to handle, especially for use in hand calculations or in providing insight into the oscillator's behavior. On the other hand, we need to know what errors are introduced when using the easier-to-handle approximate equation (4). In sections 4 and 5, we will point out when the presence of $\theta$ in the right-hand side makes a difference and when not. In order to provide a rigorous foundation for these results, we first discuss the averaging transformation as a tool to solve both equations (1) and (4).

\section{SOLVING THE PHASE EQUATIONS US- ING AVERAGING}

Both the differential equations (1) and (4), modeling the oscillator's phase behavior, belong to the more general class of ordinary differential equations (ODEs) described by

$$
\frac{d \mathbf{p}}{d t}=\epsilon \sum_{k=1}^{K} \mathbf{h}_{k}(\mathbf{p}, t)
$$

\footnotetext{
${ }^{1}$ More precisely, $v(t)$ is the inner product of the PPV and an input vector describing the way the perturbations due to $n(t)$ enter the circuit equations [3].

${ }^{2}$ For computation of the ISF, we refer to the direct measurement of the ISF by applying impulse responses [6].
}

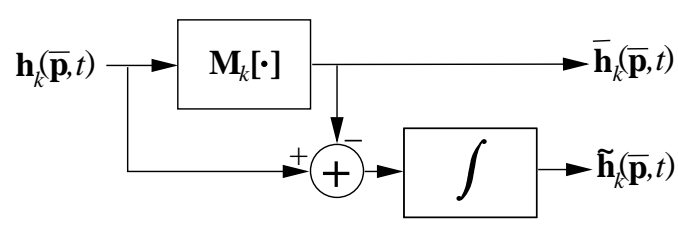

Figure 1: Constructing the averaging transformation.

where $\mathbf{p}(t) \in \mathbb{R} \rightarrow \mathbb{R}^{P}$. When dealing with the oscillator phase equations (1) and (4), $\mathbf{p}(t)=[\theta(t)]$. The set of ODEs (5) can efficiently be dealt with using averaging $[2,5]$. Averaging relies on the fact that solutions of (5) will only vary substantially on a time scale $t / \epsilon$. On the time scale $t, \mathbf{p}(t)$ can be treated as a constant (or as a stationary process when noise is involved). This allows to separate fast-varying, steady-state, and slow-varying, transient, processes.

Technically, averaging involves the construction of a transformation of variables $\mathbf{p} \rightarrow \overline{\mathbf{p}}$, determined by

$$
\mathbf{p}=\overline{\mathbf{p}}+\epsilon \sum_{k=1}^{K} \tilde{\mathbf{h}}_{k}(\overline{\mathbf{p}}, t)+O\left(\epsilon^{2}\right)
$$

This averaging transformation is chosen such that (a) the transformed ODEs are easier to solve than the untransformed ones, and (b) $\tilde{\mathbf{h}}(\overline{\mathbf{p}}, t)$ remains $O(1)$, i.e. bounded, over the time interval of interest. Both conditions are satisfied for

$$
\tilde{\mathbf{h}}_{k}(\overline{\mathbf{p}}, t)=\int\left(\mathbf{h}_{k}(\overline{\mathbf{p}}, t)-\mathbf{M}_{k}\left[\mathbf{h}_{k}\right](\overline{\mathbf{p}}, t)\right) d t
$$

with $\overline{\mathbf{p}}$ being treated as a constant. Here, the operator $\mathbf{M}_{k}[\cdot]$ is some suitable, linear, averaging operator. Selecting an averaging operator is a degree of freedom which can be used to optimize the properties of the transformed (averaged) set of ODEs. Some appropriate choices are

$$
\begin{aligned}
& \mathbf{M}_{k}\left[\mathbf{h}_{k}\right]=\frac{1}{T} \int_{t-T / 2}^{t+T / 2} \mathbf{h}_{k}(\overline{\mathbf{p}}, \tau) d \tau \\
& \mathbf{M}_{k}\left[\mathbf{h}_{k}\right]=\frac{1}{T} \frac{\sin (\pi t / T)}{\pi t / T} \otimes \mathbf{h}_{k}(\overline{\mathbf{p}}, t)
\end{aligned}
$$

with $\otimes$ denoting the convolution operator. Here, (8) is the classical averaging operator [2] and (9) is the ideal low-pass filter over the frequency range $f=\left[-\frac{1}{2 T}, \frac{1}{2 T}\right]$. The construction of the averaging transformation (6) is illustrated in Fig. 1. Inserting the transformation (6) into the ODEs (5) shows $\overline{\mathbf{p}}(t)$ to satisfy

$$
\frac{d \overline{\mathbf{p}}}{d t}=\epsilon \sum_{k=1}^{K} \mathbf{M}_{k}\left[\mathbf{h}_{k}\right](\overline{\mathbf{p}}, t)+O\left(\epsilon^{2}\right)
$$

It is now easily seen that, with the averaging operator $M_{k}[\cdot]$ given by either (8) or (9), $\overline{\mathbf{p}}(t)$ contains almost all of the low-frequency content of $\mathbf{p}(t)$ while $\tilde{\mathbf{h}}_{k}(\overline{\mathbf{p}}, t)$ only contains high-frequent components. This yields the desired separation of fast-varying and slowvarying behavior.

Results from the theory of stochastic processes [5] ensure that (6) and (10) are valid for $\mathbf{h}_{k}(\overline{\mathbf{p}}, t)$ being either a deterministic or a stochastic process. For example, if, for constant $\overline{\mathbf{p}}, \mathbf{h}_{k}(\overline{\mathbf{p}}, t)$ is a Gaussian, $T$-periodic cyclostationary noise source, then results from cyclostationary noise theory $[11,12]$ show that, for $\mathbf{M}_{k}[\cdot]$ being the ideal low-pass filter defined in equation (9), $\overline{\mathbf{h}}(\overline{\mathbf{p}}, t)=$ $\mathbf{M}_{k}\left[\mathbf{h}_{k}\right](\overline{\mathbf{p}}, t)$, is a Gaussian, stationary noise process with its au- 
tocorrelation determined by

$$
\bar{\Phi}_{k}(\overline{\mathbf{p}}, \tau)=\frac{\sin (\pi \tau / T)}{\pi \tau} \otimes \frac{1}{T} \int_{-T / 2}^{T / 2} \Phi_{k}\left(\overline{\mathbf{p}}, t+\frac{\tau}{2}, t-\frac{\tau}{2}\right) d t
$$

Here,

$$
\Phi_{k}\left(\overline{\mathbf{p}}, t_{1}, t_{2}\right)=E\left\{\mathbf{h}_{k}\left(\overline{\mathbf{p}}, t_{1}\right) \mathbf{h}_{k}^{*}\left(\overline{\mathbf{p}}, t_{2}\right)\right\}
$$

is the autocorrelation of the cyclostationary input noise source. This reduction from cyclostationary to stationary noise processes typically greatly facilitates further analysis.

This concludes our treatment of the averaging transformation. In the sections that follows, it will be applied in solving both the equations (1) and (4) when dealing with oscillator phase noise and injection locking. Studying the averaged equations will reveal the similarities and differences in the results obtained using either the exact and the approximate model.

\section{PHASE NOISE ANALYSIS}

In dealing with oscillator phase noise, i.e. the behavior of the oscillator phase when subjected to a noisy input $n(t)$, we first study the results obtained from the exact phase equation (1) presented in [3]. This is accomplished using the apparatus developed in section 3. Using averaging, we will separate the slow-varying from the fast-varying components. These slow-varying components, containing the most essential part of the phase behavior, are then compared to the ones obtained from the approximate phase equation (4).

Let us now assume $n(t)$ to be a (Gaussian) stationary noise source with its autocorrelation equal to

$$
\Phi(\tau)=E\left\{n\left(t+\frac{\tau}{2}\right)\left(t-\frac{\tau}{2}\right)\right\}
$$

We furthermore impose either that $\Phi(\tau) \rightarrow 0$ on the scale $T / \epsilon$ or that the spectrum of $\Phi(\tau)$ is mainly contained within the frequencyband $[-1 / 2 T, 1 / 2 T]$ where $T$ is the oscillation period. Equations (10) and (11) in section 3 then learn that the solution of (1) is, up to zero-th order in $\epsilon$, equivalent to that of the averaged equation

$$
\frac{d \bar{\theta}}{d t}=\epsilon \bar{n}(t)
$$

Here, $\bar{n}(t)$ is a (Gaussian) stationary noise source with its autocorrelation determined by

$$
\begin{aligned}
\bar{\Phi}(\tau) & \approx \frac{1}{T} \int_{-\frac{T}{2}}^{\frac{T}{2}} v\left(t+\bar{\theta}+\frac{\tau}{2}\right) v\left(t+\bar{\theta}-\frac{\tau}{2}\right) \Phi(\tau) d t \\
& \approx \frac{1}{T} \int_{-\frac{T}{2}}^{\frac{T}{2}} v\left(t+\frac{\tau}{2}\right) v\left(t-\frac{\tau}{2}\right) \Phi(\tau) d t
\end{aligned}
$$

It is observed that, due to the periodicity of $v(t)$, the dependence on $\theta$ of the right-hand side of equation (14) disappears. This is however only valid if $n(t)$ is a stationary noise source, making the autocorrelation $\Phi(\tau)$ independent of $t$ and hence making the transition from equation (15) to (16) possible. It can be shown that further results, for both white and colored noise sources, obtained using equations (14) and (16) are identical to the ones in $[4]^{3}$. We hence won't go into depth, but refer to [4] for further details.

\footnotetext{
${ }^{3}$ Note that in [4], these results were obtained through an alternative approach using the construction of the (modified) Fokker-Planck equation. Using the averaging transformation, this often complicated equation is avoided providing simpler ways to deal with noise in oscillators, both white and colored.
}

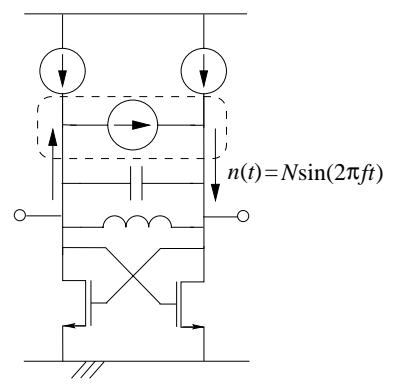

Figure 2: Harmonic oscillator being injected with a sine wave.

A similar analysis, but now starting from the approximate equation (4), also yields the averaged equation (14) with

$$
\bar{\Phi}(\tau) \approx \frac{1}{T} \int_{-\frac{T}{2}}^{\frac{T}{2}} \Gamma\left(t+\frac{\tau}{2}\right) \Gamma\left(t-\frac{\tau}{2}\right) \Phi(\tau) d t
$$

When $v(t)=\Gamma(t)$, this is identical to the result in equation (16). Remember, however, that the latter was only valid for stationary noise input sources.

As a conclusion, we can state that, as far as stationary noise input sources are concerned, there is, up to zero-th order in $\epsilon$, no difference in the results obtained from the exact model (1) and the approximate model (4). It is hence safe to use either of the models presented in [3] and [6] as a starting point for phase noise analysis.

\section{INJECTION LOCKING}

As was mentioned in the previous section, the condition for both the exact and the approximate model to yield the same result, is the stationarity of $n(t)$. If this condition no longer holds, results predicted by both models start to diverge. This can clearly be seen when investigating an oscillator's injection locking behavior $[1,7]$. Consider for example the setup shown in Fig. 2. Here, a harmonic oscillator is injected with a sine wave with frequency $f$. A phenomenon known as injection locking, makes that the oscillator locks both in phase and frequency to this sine wave, this for frequencies $f$ close to the oscillator's free-running frequency $f_{0}=1 / T$. It is the principle underlying a number of phase and frequency modulators. It is also the principle behind systems of coupled oscillators, involving mutual injection locking. In what follows, we investigate what both the exact model (1) and the approximate model (4) tend to predict for this locking phenomenon. We do so for the harmonic oscillator example shown in Fig. 2.

It can be shown that, roughly, the output voltage of a harmonic oscillator is given by

$$
V_{o s c}(t)=A \cos \left(2 \pi f_{0}(t+\theta(t))\right)
$$

with $A$ the oscillation amplitude and $\theta(t)$ the instantaneous phase (in seconds). When subjected to a sinusoidal input current $n(t)=$ $N \sin (2 \pi f t)=N \cos (2 \pi f(t-T / 4))$ the oscillator's phase behavior can be modeled using either (1) or (4) with

$$
v(t)=\Gamma(t)=-V \sin \left(2 \pi f_{0} t\right)
$$

Using the same procedure as in the previous section, we now consider the results predicted by both the exact and the approximate model by comparing the associated averaged equations as determined by (10). In order to keep the analysis simple, we assume that $f=f_{0}$. Results are however easily extended towards the case where $f=f_{0}+\Delta f$. 


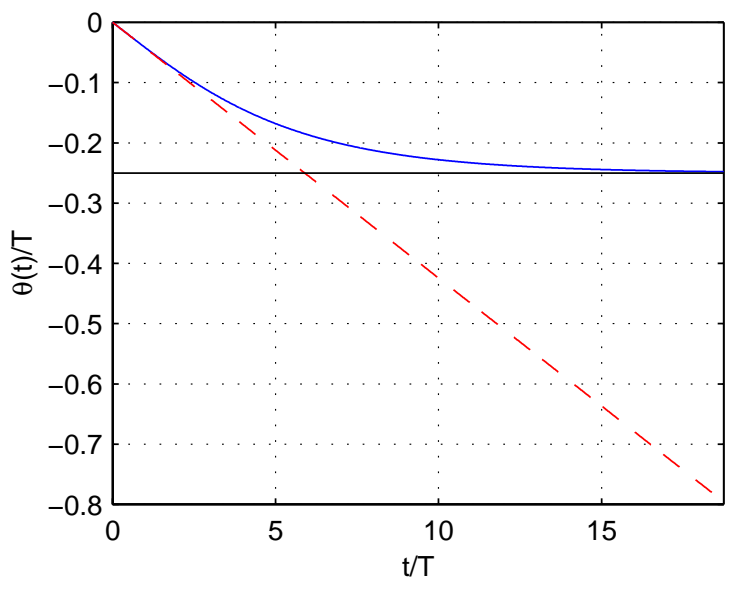

Figure 3: Behavior of the phase $\theta(t)$ of a harmonic oscillator injected with a sinusoidal current. The results obtained from the model (20), indicated by the blue solid line, predicts phase locking, while the results obtained from the model (23) indicated by the red dashed line, predicts a frequency shift.

Use of the exact model (1) yields

$$
\frac{d \theta}{d t}=-\epsilon N V \sin \left(2 \pi f_{0}(t+\theta)\right) \sin \left(2 \pi f_{0} t\right)
$$

resulting in the zero-th order averaged equation

$$
\frac{d \bar{\theta}}{d t}=-\epsilon \frac{N V}{2} \cos \left(2 \pi f_{0} \bar{\theta}\right)
$$

This implies that, for $\bar{\theta}(0)=0$, the phase will start to drop, initially at a rate $-\epsilon N V / 2$, until it reaches $\bar{\theta}=-T / 4$, a quarter of the oscillator period. It is easily verified that this point is a stable equilibrium of equation (21). In this equilibrium point, the oscillator output voltage becomes

$$
V_{o s c}(t)=A \cos \left(2 \pi f_{0}\left(t-\frac{T}{4}\right)\right)=A \sin \left(2 \pi f_{0} t\right)
$$

The model hence predicts that the phase of the output voltage locks onto that of the input current source, as it is expected and observed to do. The solid line in Fig. 3 depicts this locking behavior for $\bar{\theta}(t)$ starting from $\bar{\theta}(0)=0$.

On the other hand, the approximate model (4), states that the phase behavior is governed by

$$
\frac{d \theta}{d t}=-\epsilon N V \sin \left(2 \pi f_{0} t\right) \sin \left(2 \pi f_{0} t\right)
$$

This results in the zero-th order averaged equation

$$
\frac{d \bar{\theta}}{d t}=-\epsilon \frac{N V}{2}
$$

implying that the phase $\bar{\theta}(t)$ would drop at a rate $-\epsilon \frac{N V}{2}$, but now indefinitely. Stated otherwise, the approximate model predicts a change in frequency, or

$$
V_{o s c}(t)=A \cos \left(2 \pi\left(1-\epsilon \frac{N V}{2}\right) f_{0} t\right)
$$

which is not quite the locking behavior that we were expecting. The dashed line in Fig. 3 shows the difference with the locking behavior as predicted by (21). Note that at first, near $t=0$, the predicted phases are the same. However, when $\bar{\theta}$ becomes too large to be neglected in the right-hand side of the averaged exact phase equation (21), results start to diverge.

As a conclusion, we can state that when the input sources are no longer stationary, results obtained from the exact and the approximate model start to diverge, with the exact model predicting the correct results. This implies that the approximate model is, for example, not accurate enough to analyze injection locking nor related issues like the behavior of phase differences within sets of coupled oscillators.

\section{EXPERIMENTAL RESULTS}

The conclusions above were verified through numerical experiments on the harmonic oscillator setup shown in Fig. 2. In a first step, we studied the characteristics of the output phase due to a white input noise source $n(t)$. Results as predicted using the exact and the approximate model were compared. In a second step, we simulated a current-injected oscillator to verify whether locking occurs.

In order to verify our conclusions concerning results for stationary input sources, we solved both (1) and (4) for a stationary, Gaussian, white input noise source $n(t)$ and for the PPV $v(t)$ equal to the ISF $\Gamma(t)$. This PPV/ISF was taken to be the one of the harmonic oscillator in Fig. 2. The stochastic behavior of the output phase $\theta(t)$ was characterized by means of its time-varying variance

$$
\sigma_{\theta}^{2}(t)=E\left\{\theta(t)^{2}\right\}
$$

which was computed by solving both models for 500 different noise samples. The resulting standard deviations are shown in Fig. 4. As can be seen, both models clearly predict the same behavior for $\sigma_{\theta}^{2}(t)$ which increases linearly with time. This demonstrates the fact that, for phase behavior due to stationary input sources, it doesn't really matter which model, either the exact one (1) or the approximation (4), is solved.

On the other hand, when $n(t)$ is no longer stationary, we have seen that the models (1) and (4) predict largely different results. According to the exact model (1), the oscillator output voltage would lock onto the sine wave while according to the approximate model (4), the oscillator voltage would not lock but a frequency shift would occur. Although injection locking is a widely known phenomenon $[1,7]$, we still ran some simulations to verify that the locking behavior, as predicted by the exact model, indeed occurs. Fig. 5 shows the (normalized) behavior of a harmonic oscillator (solid line) injected with a sine wave (dashed line). The oscillator startup conditions were chosen such that $\theta(0)=0$. As can be seen from the upper figure, the sine wave makes the oscillator phase to move towards $\theta=-\pi / 2$. In order to show that this move is not due to a constant frequency shift, the startup and final oscillator voltage are shown in the lower figures of Fig. 5. These figures show that, while for startup $\theta=0$, after sufficiently long time, the oscillator phase locks firmly onto $\theta=-\pi / 2$, i.e. the oscillator output voltage locks onto the injected sine wave. This clearly demonstrates the correctness of the predictions made by the exact model equation (1), also for non-stationary input signals. Calculations made using the approximate model equation (4), however, break down. It should hence not be used for analyzing the phase behavior of oscillators subjected to non-stationary input signals.

\section{CONCLUSIONS}

This paper has investigated the similarities and differences between two widely publicized models for describing oscillator phase behavior, an exact one presented in [3] and an approximate one presented in [6]. It was demonstrated that, for stationary input sources, 


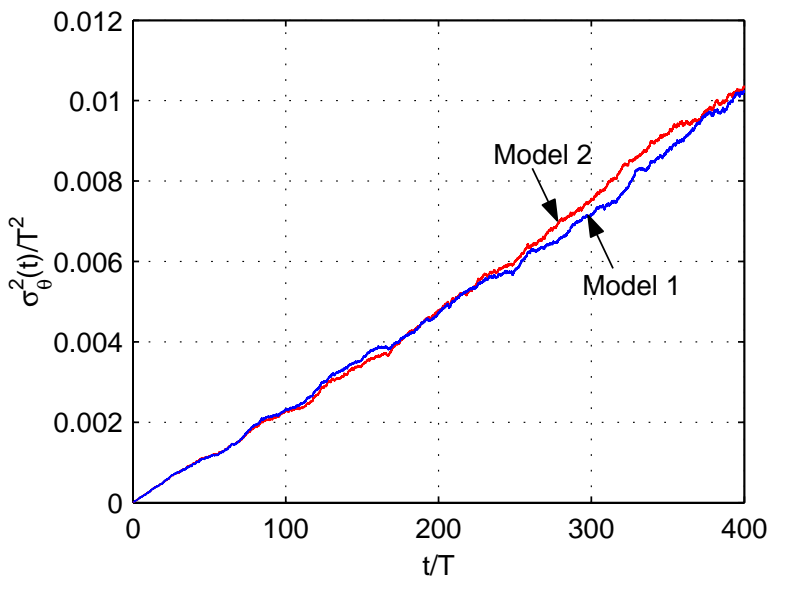

Figure 4: Variance $\sigma_{\theta}^{2}(t)$ of harmonic oscillator phase noise $\theta(t)$ caused by a white noise current disturbance. Here, the lower (blue) line indicates the results obtained from the first model (1), while the upper (red) line indicates the results obtained from the second model (4).
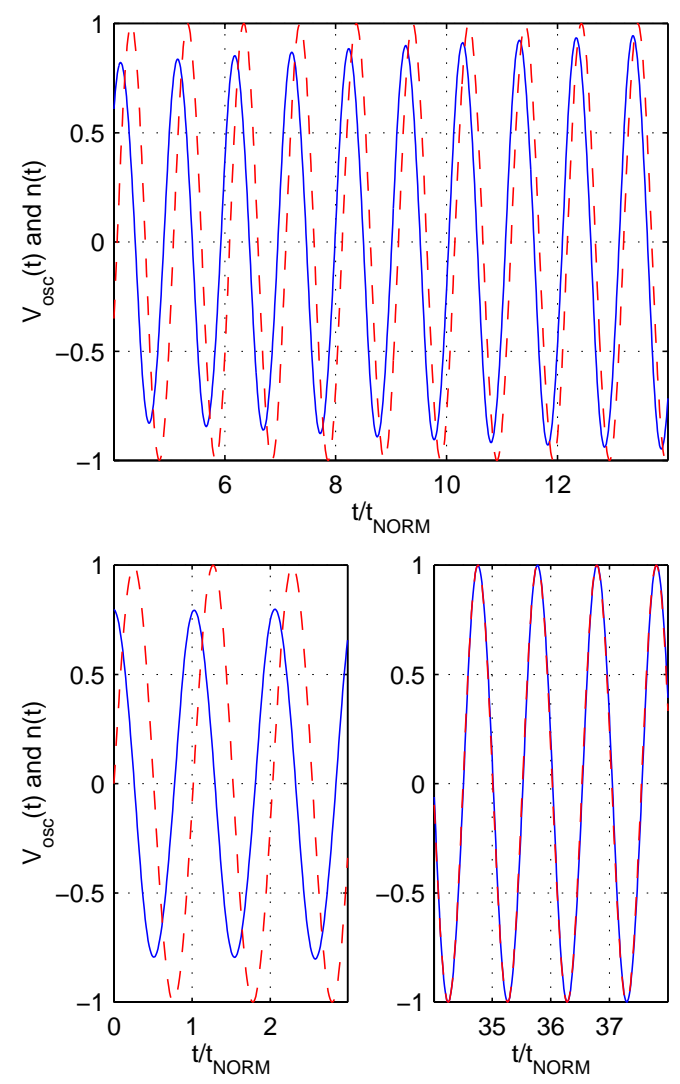

Figure 5: Locking of a harmonic oscillator due to current injection. The upper figure shows the transition from unlocked to locked. The lower figures respectively show the starting conditions, where the (normalized) oscillator signal (solid blue line) and injected current (dashed red line) are $90^{\circ}$ out of phase, and the settled behavior, where oscillator and injected current are firmly in lock. both models predict, up to zero-th order, the same stochastic characteristics for the output phase. On the other hand, when the input source is no longer stationary, results diverge. This was demonstrated for the injection locking behavior of a harmonic oscillator. Here, the exact model is capable of predicting this behavior, while the approximate model breaks down. From a theoretical point of view, these results were established using averaging, which turns out to be a powerful method to deal with the type of equations arising when analyzing oscillator phase behavior. Our results were verified through numerical simulations.

\section{REFERENCES}

[1] R. Adler, "A study of locking phenomena in oscillators", Proc. IRE, vol. 34, pp. 351-357, June 1946

[2] N.N Bogoliubov and Y.A. Mitropolsky, Asymptotic methods in the theory of nonlinear oscillations, Hindustan Publishing Corp., India, 1961

[3] A. Demir, A. Mehrotra and J. Roychowdhury, "Phase Noise in Oscillators: A Unifying Theory and Numerical Methods for Characterization", In IEEE Trans. Circ. and Syst.-I, vol. 47, no. 5, pp. 655-674, May 2000

[4] A. Demir, "Phase Noise in Oscillators: DAEs and Colored Noise Sources", In Proc. IEEE ICCAD, pp. 170-177, 1998

[5] M.I. Freidlin and A.D. Wentzell, Random perturbations of dynamical systems, 2nd. edition, Springer-Verlag, 1998

[6] A. Hajimiri and T.H. Lee, "A General Theory of Phase Noise in Electrical Oscillators", In IEEE J. Solid-State Circ., vol. 33, no. 2, pp. 179-194, February 1998

[7] M.T. Jezewski, "An Approach to the Analysis of Injection-Locked Oscillators", In IEEE Trans. Circ. Syst., vol. 21, no. 3, pp. 395-401, May 1974

[8] F.X. Kaertner, "Analysis of White and $f^{-\alpha}$ Noise in Oscillators", In Int. J. Circuit Theory Appl., vol. 18, pp 485-519, 1990

[9] T.H. Lee and A. Hajimiri, "Oscillator Phase Noise: A Tutorial”, In IEEE Journ. Solid-State Circ., vol. 35, no. 3, pp. 326-336, March 2000

[10] O. Narayan and J. Roychowdhury, "Multi-Time Simulation of Voltage-Controlled Oscillators", In Proc. IEEE DAC, Session 36, 1999

[11] J. Roychowdhury, D. Long and P. Feldmann, "Cyclostationary noise analysis of large RF circuits with multitone excitation”, In IEEE J. Solid-State Circ., vol. 33, pp. 324-335, March 1998

[12] T. Ström and S. Signell, "Analysis of Periodically Switched Linear Circuits", In IEEE Trans. on Circ. Syst., vol. 24, no. 10, pp. 531-541, October 1977 